\title{
Aircraft to Operations Communication Analysis and Architecture for the Future Aviation Environment
}

\author{
Huw W|hitworth \\ Centre for Autonomous and Cyber \\ Physical Systems \\ Cranfield University \\ Bedford, United Kingdom \\ h.t.whitworth@cranfield.ac.uk \\ Julia Jiggins \\ Thales UK \\ Crawley, United Kingdom \\ julia.jiggins@uk.thalesgroup.com
}

\author{
Saba Al-Rubaye \\ Centre for Autonomous and Cyber \\ Physical Systems \\ Cranfield University \\ Bedford, United Kingdom \\ s.alrubaye@cranfield.ac.uk \\ Nigel Silverthorn \\ Thales $U K$ \\ Crawley, United Kingdom \\ nigel.silverthorn@uk.thalesgroup.com
}

\author{
Antonios Tsourdos \\ Centre for Autonomous and Cyber \\ Physical Systems \\ Cranfield University \\ Bedford, United Kingdom \\ a.tsourdos@cranfield.ac.uk \\ Karim Thomas \\ Thales, $U K$ \\ Crawley, United Kingdom \\ karim.thomas@uk.thalesgroup.com
}

\begin{abstract}
Fifth Generation (5G) systems are envisaged to support a wide range of applications scenarios with varying requirements. 5G architecture includes network slicing abilities which facilitate the partitioning of a single network infrastructure on to multiple logical networks, each tailored to a given use case, providing appropriate isolation and Quality of Service (QoS) characteristics. Radio Access Network (RAN) slicing is key to ensuring appropriate QoS over multiple domains; achieved via the configuration of multiple RAN behaviors over a common pool of radio resources. This Paper proposes a novel solution for efficient resource allocation and assignment among a verity of heterogeneous services, to utilize the resources while ensuring maximum QoS for network services. First, this paper evaluates the effectiveness of different wireless data bearers. Secondly, the paper proposes a novel dynamic resource allocation algorithm for RAN slicing within 5G New Radio (NR) networks utilising cooperative game theory combined with priority-based bargaining. The impact of this work to industry is to provide a new technique for resource allocation that utilizes cooperative bargaining to ensure all network services achieve minimum QoS requirements - while using application priority to reduce data transfer time for key services to facilitate increase turn around time at the gate.
\end{abstract}

Keywords-5G, Network Slicing, Game Theory, RAN, Aviation, Aircraft Communications.

\section{INTRODUCTION}

There are three key categories for aviation operational data - Air Traffic Control (ATC) and Airline Operations Centre (AOC) and InFlight Entertainment (IFE) communications. For this project we are removing ATC data from the communications stream for two reasons. Firstly, the safety constraints on the messages for safety critical flight operation. Secondly, Ehammer Et Al. [1] show that ATC data comprises a smaller segment of traffic passing through the aviation network compared to AOC within relative zones. AOC data is that which is required for the initiation, continuation, diversion or termination of flight for safety, regulatory or efficiency reasons [2]. It comprises voice and data communications between aircraft and their operating agencies:

- Airline Operational Control Centre, Pilots and Ground Crew.

- Ground Services and Organisations, Ground handling, Fuelling and Maintenance.

- Engine Manufacturers

Over time, the aviation industry has seen an increase in AOC traffic as aircraft fleets have grown and evolved, deployment of new aircraft has led to an increase if transmitted engine and maintenance data. The traffic increase has been compounded by some airlines using the AOC stream to communicate with flight crews and to report specific operational information. Currently ATC and AOC traffic is transmitted through a single VDL system using the same equipment; Shared use of a limited service has resulted in an increase in failed transmissions due to collisions [3]. Given this, within the aviation environment there is a need for ATM modernization and a paradigm shift away from legacy technology such as VHF and VDLM2 towards broad spectrum, high data rate low latency infrastructure.

The current systems employed within the ATM framework do not compare with the current state of the art technology such as Fifth Generation $(5 \mathrm{G})$ or even the previous generation of data links $(4 \mathrm{G}$, IEEE 802.11ac...). Regards VDLM2, a recent proposal from SESAR [4] stated that it has the same inherent capability as SMS. As connectivity becomes more prevalent throughout the aviation ecosystem the connected aircraft concept is closer than ever. The implementation of an IP driven flight-deck with real time systems will facilitate a revolution in operational efficiency. Current air traffic growth is approximately $6 \%$ per annum [5]. Early predictions of increased traffic growth state that aeronautical spectrum resources will be insufficient to accommodate the predicted growth due to Radio Frequency (RF) saturation expected in high-density aviation environments [6] such as Central Europe or West/East Coast America - indicating the need for a system that is scalable and future proof. A plausible solution is the implementation of a communication medium to relieve the burdening AOC data from the VHF spectrum; relieving congestion from the legacy systems for the time being.

The IFE datalink services are those which facilitate the transfer of media contents to the aircraft and/or provide regular updates to existing services [7]. IFE services broadly fall into the following categories:

- $\quad$ Entertaining Passengers

- Inform Passengers

- Support Cabin Crew

In this paper we have proposed a novel algorithm based on cooperative game-theory that solves the resource allocation optimization and scheduling, and network QoS profile problems though a systematic selection of control variables (Resource Blocks). The model we have derived utilizes the strategic cooperation between services, slices and resource blocks. We believe these algorithms could be used to maximize efficiency in 5G Ground/Ground networks and inter-slice resource coordination.

This paper is organized as follows: An overview of critical infrastructure and technology used at the airport surface is given in Section II. System design and development of RAN architecture is Section III. System Modeling and Game Theory algorithms are derived in section IV. Concluding remarks are given in Section V. 


\section{TECHNICAL ASPECTS OF AIRPORT SySTEMS}

\section{A. Current ATC/ATM communication deployment:}

Currently, there is no uniform connectivity framework or infrastructure deployed for Aircraft-Airline-Airport interaction. The current state of the art platforms is based on either Fifth Generation (5G) Cellular technology; or Wi-Fi solutions. Despite the benefits of state-of-the-art technologies, legacy technology such as Very High Frequency (VHF), Aeronautical Communication and Reporting System (ACARS) and Satellite Communication (SATCOM) systems are deployed for Air Traffic Control (ATC) and Air Traffic Management (ATM) purposes due to the longstanding reliability, security, and availability requirements.

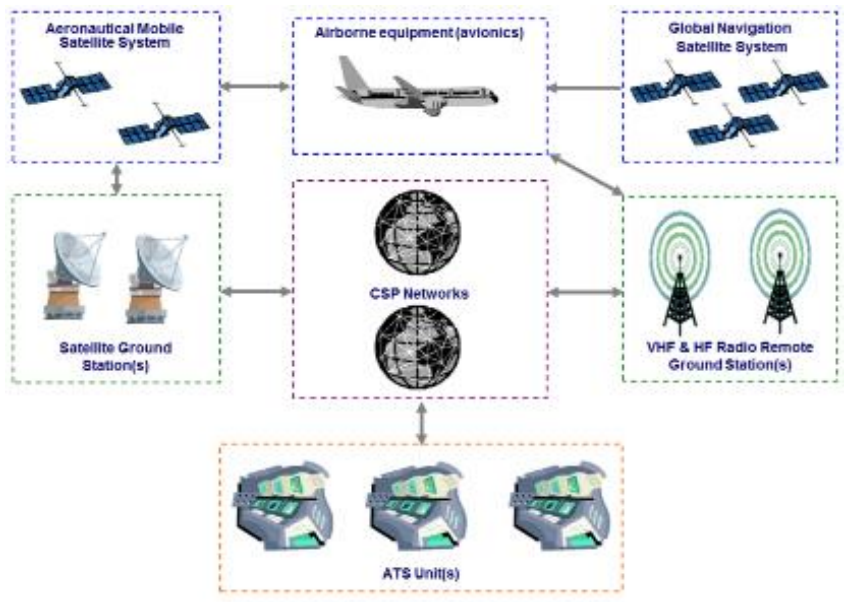

Figure 1 Overview of ICAO ATS- Aircraft Data Links [4]

There is a high demand for seamless radio communications link need to be deployed quickly to enable the flow of data services using air-to-air (A2A) and air-to-ground (A2G) links. With the rapid development of data communications, the International Telecommunication Union (ITU) predicts that the launch of the 5G networks in the near future will play a significant role in the airspace domain and pave ways for novel applications. Using this technology, the aircraft communication systems will reach a speed of $100 \mathrm{~GB} / \mathrm{s}$ with a data rate capacity of almost 1000 times wider than the existing system [8].

Figure 1 shows the overview of data links from Air Traffic Services (ATS) units to the aircraft including necessary sub networks. However, the current and expected increase of aviation traffic coupled with the heavily congested and limited VHF spectrum indicates it will not be able to keep pace with the rapidly growing air traffic and future high-speed data needs. Satellite Communication (SATCOM) is proposed alternative to VHF; however, SATCOM solutions can be prohibitively expensive and have been shown to suffer poor connectivity at airport surfaces due to interference and buildings. From this we can conclude that legacy technology such as VHF, HF and SATCOM may be suited for high altitude cruising data exchanges; they are not suitable for Air Traffic Management Services (ATMS) surface data exchanges.

The ATM are all those systems that assist aircraft departure, Transit and Landing from/at an aerodrome. Eurocontrol COCR 2.0 determines that by the 'Future Communications System' (FCS) (>2035) infrastructure will continue to consist of Human Machine Interfaces (HMI), voice switches, flight data processing systems, ground communication systems, routers, networks, radio ground stations and communication end to end systems combined in an endto-end chain. As such they must meet performance and safety requirements for voice and data applications.

\section{B. Critical Infrastucture:}

Ground level critical infrastructure can be split in to two streams: Air Traffic Control (ATC) and Airline Operational Control (AOC) services. ATC services are those such as clearances and critical communications for the facilitation of establishing control and flight safety. Whereas AOC provides for the continual effectiveness of airspace operation. AOC services comprise the vast majority of interaction between the Airline Operational Control Centre (AOCC), 3rd-party company and airport operational personnel. It is a requirement of the Federal Aviation Authority (FAA) that ATC and AOC traffic be segregated across all networks [9]. There are two primary methods of assuring data segregation: The implementation of multiple independent networks, or the use of a single network using traffic-handling features available within the communication medium profile.

In order to separate ATC and AOC through the independent network model two or more networks need to be implemented. This full separation of traffic presents some challenges:

1. The requirement for split spectrum allocations in order to facilitate multiple networks.

2. An inherent inflexibility for resource allocation between ATC/AOC domain based on network stress.

3. Increased Potential for Link Interference from multiple network operation.

ATM modernization requires a paradigm shift away from voice, towards digital data communication. Increased and more complex information exchange between controllers and pilots determine the need more modern communication technology. Currently, for Air Traffic Control and Critical Communications, VHF, ACARS and SATCOM are commonplace. However, publications from EUROCONTROL, SESAR and the FAA [10], [11] present the deployment of AeroMACS as the new-generation airport datalink to cope with increased volumes of data being exchanged at the airport surface. [11] [13] AeroMACS offers to support ATC and AOC services at the airport surface for critical operation; It provides higher throughput for airport surface communication while relieving some of the congestion felt on the VHF bands. Employing IEEE 802.16 allows industry to employ adaptive modulation; using unicast transport connections (service flows) allows each service to set particular QoS parameters such as: Latency, Jitter, and Throughput Assurance. Furthermore, IEEE 802.16e service flows allow the segregation of ATC/AOC traffic implemented through the deployment of multiple Core Service Networks.

For Air Traffic Management communication many airports still use legacy technology use as VHF, ACARS and SATCOM. However, many of these systems have inherent issues such as the over utilization and congestion of the VHF spectrum and SATCOM poor surface connectivity in the urban environment [14]. To counter this many airports are using modern commercial systems such as Cellular or Wi-Fi however these links are prohibited for ATC deployment or any other safety critical data exchange.

The current state of the art for airport surface communication is AeroMACS. Operating as the international standard broadband datalink for safety and regulatory flight communication exchanges at the airport surface. Based off IEEE 802.16 standard 4G technology operating in the $\mathrm{C}$ band frequency. AeroMACS support for a wide range of applications (Voice, Video, Data) make it an ideal solution coupled with its inherent use of priority levels and security capability to ensure the segregation of ATC/AOC communication. A main drawback of AeroMACS is its low data rate of $\sim 9.2 \mathrm{MB} / \mathrm{s}$. 
In the following segment we evaluate existing, practical wireless technologies ranging from 802.11 standard Wi-Fi to $5 \mathrm{G}$ cellular. We also explore and explain technology enablers such as network slicing and network virtualization.

\section{802.11 Standard Wi-Fi}

Since $1997 \mathrm{Wi}-\mathrm{Fi}$ standards have been in a state of constant evolution in order to provide users with greater throughput, coverage and bandwidth while ensuring reduced interference and latency. The following section provides a functional level overview of the main Wi-Fi standards:

\subsection{1ac:}

- Wi-Fi 5 (802.11ac) standard specifies up-to 8 streams (8X8:8) and uses a channel width of 80 to $160 \mathrm{MHz}$. In order to reduce interference with other stations or network participants, the IEEE802.11ac incorporates standardized beamforming. Ieee802.11ac provides a throughput of 1 Gbps using a high-density modulation scheme (256QAM).

- $\quad 802.11$ ac standard operates within the $5 \mathrm{GHz}$ band and is able to provide coexisting mechanisms for $11 \mathrm{n}$ and $11 \mathrm{ac}$. Several papers (inc. ICAO) advised when using IEEE802.11ac channels as domain assignment is dependent on the regulatory body of a set geographical location (I.E. Civil Aviation Authority, UK \& Federal Aviation Authority, USA).

\section{$802.11 a x$}

- Wi-Fi 6 (802.11ax) is the subsequent improvement on $11 \mathrm{ac}$. Operating in both the $2.5 \mathrm{GHz}$ and $5 \mathrm{GHz}$ frequency spectrum, Wi-Fi 6 has been designed to support dense deployment. Subcarrier spacing has been reduced for 11.ax in order to sub divide the channels into a more granular setup has divided the spacing by 4 in to $78 \mathrm{KHz}$. This granularity creates a layer of flexibility. The symbol time is the time used on each carrier when transmitting. As we can see this has been increased from 4 to 16 microseconds.

- Frequency multiplexing options have been increased from $\operatorname{OFDM}(\mathrm{HT})$ all of which are supported in 11.ax along with OFDMA. 11.ax has also increased to 1024 QAM which equates to 10 bits per symbol meaning a potential $25 \%$ increase in data link throughput. 11.ax piggybacks on MU-MIMO with an LTE cellular base station technology - Orthogonal Frequency Division Multiple Access (OFDMA). This allows each MU-MIMO stream to be split in four additional streams, boosting the effective bandwidth per user by four times.

It should be noted that often there is overlap on a given frequency band; as such operating parameters must be chosen with care to avoid undue interference. Additionally, directional antennas may be utilized to help negate the effects of unwanted interference in certain scenarios.

\section{Fifth Generation (5G)}

The Fifth-Generation network is the next paradigm shift upon Fourth Generation (4G) Cellular Technology. Recognizable by its decided increase in throughput, bandwidth and base station density $5 \mathrm{G}$ is set to transform communication infrastructure. Within the $5 \mathrm{G}$ systems, $3 \mathrm{GPP}$ release 15 we have identified two generic use cases applicable to this work :

- $\quad$ Enhanced Mobile Broad Band (eMBB); eMBB supports applications to meet user demand for high data rates and large bandwidth requires such as aviation In-Flight Entertainment (IFE) data.

- Ultra-Reliable and Low Latency Communications (URLLC); URLLC focusses on supporting low-latency transmissions of small payloads with high reliability for a range of active terminals. Applicable to mission critical data exchanges.

URLLC requires extremely low latency $(0.25-0.3 \mathrm{~ms} /$ packet $)$ and a high reliability $(99.999 \%)$ while eMBB requires high bandwidth $(>100 \mathrm{MHz}$ to support gigabit per second for high data rate peaks)[12]. Due to this, the traditional method of 'one-size-fits-all' networking may no longer be appropriate; coupled with the increase in heterogeneous networking - mmWave communications and network slicing have been revealed as key enablers of future communication systems.

\section{E. Network Slicing:}

Efficient Radio Access Network (RAN) slicing algorithms will result in benefits such as improving the network capacity utilization, avoiding service outages due to lack of resources, reducing the network traffic congestion and ensuring high QoS in terms of data rate and latency [16] [17]. The proximity of multiple slices operating on a singular infrastructure requires isolation between created elements. A key challenge within network slicing is the ability to operate as an isolated entity and therefore remain unaffected by coexisting slices [18].

An area of interest is combining network slicing with mmWave wireless networks to satisfy heterogenous network traffic demand through flexible operation within frequency bands. Slicing QoS is augmented by network virtualization (NV) which allows network operators to share common physical infrastructure in a dynamic manner. This paper examines how to serve URLLC and eMBB slices that share the same radio access resources without compromising quality of service of slice users.

The results of [19] indicate that that the introduction of RAN slicing through carrier aggregation is beneficial for delay. Lowest delay is reasonably achieved when both flows are isolated. As URLLC transmissions can be independently scheduled without delay due to the presence of eMBB packets in the queue. Furthermore, the possibility to utilize a carrier operating at lower frequencies to ensure reliable data delivery due to fewer Medium Access Control (MAC) and Radio Link Control (RLC) layer retransmissions. Therefore, the RAN slicing problem to support eMBB \& URLLC on the same RAN infrastructure becomes an optimization problem to determine the amount of resources assigned to each slice with the aim of improving radio resource utilization while satisfying the specific requirements for each slice.

\section{F. Resource Management:}

Differing slices of a RAN can share the radio resources in either a dynamic or static manner, dependent on configuration [20]. The static resource assignment operates a slice with a fixed resource. This provides a guarantee of resource allocation to slices; however, is incapable of adapting for differing demand criteria. Conversely, dynamic resource allocation refers to the ability of a system to sense change in environment allowing each slice to dynamically adjust allocated resources based on demand and priority (URLLC supersedes eMBB in transmission priority) to optimize the quality of the communication services [21][22].

\section{SYSTEM DESIGN AND DEVELOPMENT}

This paper is interested in evaluating how two different services; eMBB \& URLLC, described in section 3 can efficiently share the same radio resources when transferring data from the gate to the aircraft. 
A scenario comprised of a common radio infrastructure shared by $\mathrm{N}$ tenants is assumed. Each tenant operates a pre-generated and deployed RAN slice through the Network Slice Subnet Management Function (NSSMF) [23]. The diagram shown in Figure 2 is comprised of a gNB cell of bandwidth B divided into Physical Resource Blocks (PRB). To ensure the efficient use of radio resources and therefore the attainment of QoS parameters, the gNB utilizes RMM functions - For this paper we are focusing primarily on Admission Control and Resource Allocation.

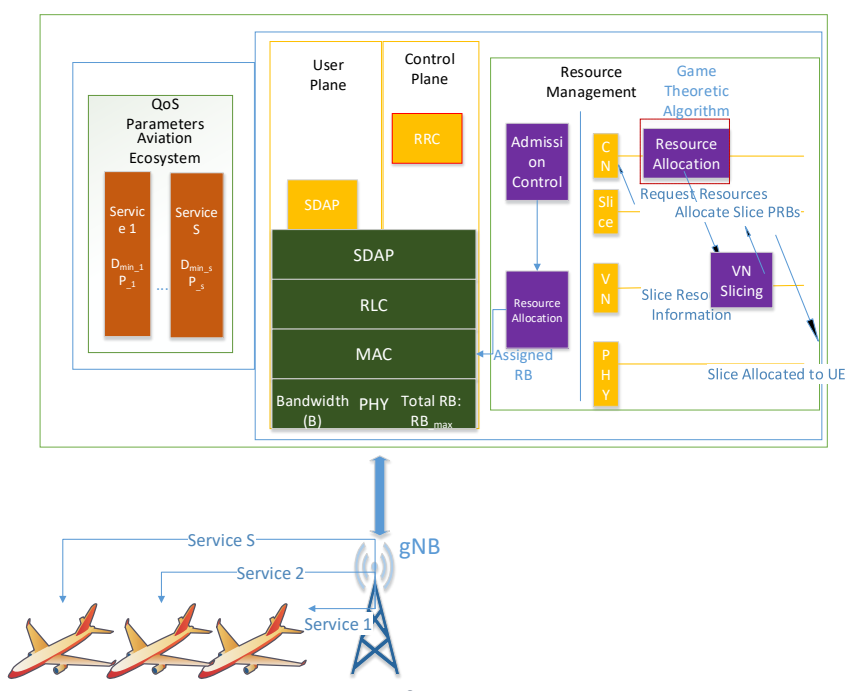

Figure 2 : High Level Diagram of gNB communications

We propose a RAN Slicing Resource Management (RSRM) algorithm which is capable of dynamically distributing bandwidth among different slices within a gNodeB to maximize resource utilization. The architectural model for RSRM is based on SDN and NFV and split in to four core segments: Resource Allocation Mechanism, Physical Resources, Virtual Network Layer and Service layer. We propose the implementation of a cooperative game theory algorithm to distribute resource blocks between coalitions of services with the objective to maximize resource utilization. Prior work has employed non-cooperative game theory[21]. However, we believe that an algorithm based on cooperative game theory will be less computationally expensive [24] and therefore more suitable to the future aviation environment.

\section{A. Modeling of Radio Access Network:}

5G uses a new technology called network slicing to provide networks that are suitable to various services in the form of varying network slices. Slices are set with requirements such as throughput, delay and reliability - network resources are allocated to slices to satisfy these requirements. To deploy the NS concept various network resources should be divided and each resource should be allocated to satisfy the requirement of that slice. The radio access network (RAN) is challenged with generating a solution that satisfies the slice requirements in a dynamic manner; without reducing the efficiency compared to when compared to finite radio resource allocation. The number of slices processed by a base station depends on the flow of incoming and outgoing UE from the BS coverage area and service usage; however, give the airport environment we shall assume a constant. Therefore, a method is necessary that flexibility allocates radio resources according to slices status.

It is an important aspect of network slicing to satisfy slice requirements. However, as RBs are a limited resource if a slice over utilizes, these resources cannot be allocated to other slices- thereby increasing the potential for loss of QoS or service outages. As such, it is necessary to only allocated the minimum number of RBs to a slice. We propose a cooperative game model (Player, Action, Payoff) that satisfies the slice requirements and minimizes the number of allocated RBs. We believe the simple algorithm proposed leads to Shapley-Value based algorithms that utilize the advantage found in the strategic cooperation between reactive-resource-block allocation and relative network slices.

\section{a) Service Layer:}

As we have previously stated the aviation ecosystem comprises multiple elements each requiring resources to service clients:

As shown in Figure 3, the operational domain of the aviation environment can be divided in to three core elements: Airlines, Airport and Aircraft. Each section is linked to the aircraft via the transmission of AOC data. A breakdown of AOC services used by the aircraft is given by Ehammer Et Al.[1]

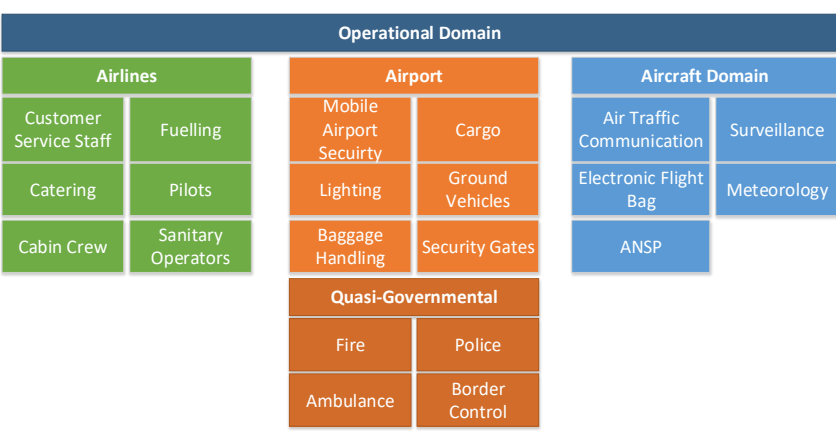

Figure 3 : Various actors requiring services and resources to transmit data within the ecosystem

AOC data is not the only exchange between the aircraft and the gate. In-Flight Entertainment IFE data refers to the entertainment available to aircraft passengers during flight. The average IFE data transfer rates are:

- $\quad$ 500GB per Month

- $\quad$ 112GB per Week

- $\quad$ 20GB per Flight

IFE is defined as its own service as with those operating under the operational domain. However, unlike many of the other services we do not assume that all flights departing will provide IFE services during flight - for example internal flights within the UK or Europe. Services are designated virtual network slices, $S=\{1,2, \ldots, S\}$. Each slice is able to provide a service to multiple UEs which is denoted as a subset of $\mathrm{S} ; \mathrm{Us}=\{1,2, \ldots, \mathrm{Us}\}$. As each slice provides a service it performs a request to the resource allocation module in the form of QoS criteria $D_{\text {Min } s}$, $D_{\text {Max } s}$ and $\mathrm{P}_{s}$ representing min/max data rates and priority for slice $S$ accordingly. Similarly, a priority system is applied to the data passing through the slices. For user $\mathrm{U}$ within slice $\mathrm{S}$ is assigned a QCI priority value.

\section{b) Virtual Network:}

Network virtualization is the partitioning or combining of network resources. Dependent on resource allocation determined by admission control the virtual network slices are assigned the resources to accommodate functionality. Figure 2 shows the flow of requests from slice to the Resource Allocation Framework (RAF) which then determines the allocation of Physical Resource Blocks (PRBs) dependent on UE QoS and relative intra- and inter-slice priority.

\section{c) Physical Resources:}

Physical Resources are those radio resources that can be utilized by the virtual network. A Resource Element (RE) is the smallest unit resource made up of one subcarrier in the frequency domain and one OFDM symbol in the time domain. A Resource Block (RB) is given as 12 consecutive subcarriers in the frequency domain of variable 
bandwidth as the bandwidth of a NR RB is given as $12 *$ Sub Carrier Spacing.

As time series allocation is often dynamic, static resource allocation will often leave idle resources in various times/locations. our model allocates the computational resources of the network in case of resource shortages by prioritizing RBs based on the type of services that are active and QoS limitations. Furthermore, our model attempts to guarantee the minimum computational resources be allocated to a service to prevent resource starvation and service failure

\section{Sheduling:}

d) QCI Based resrouce type classification \& Within our system we have adopted the 3GPP style QoS Class Identifier (QCI). Using this system, we classify services as either Guaranteed Bit Rate (GBR) or Non-GBR. Service categories are further devolved in to several QCI ranks of differing packet delay budget and rate of packet error loss. It is assumed that resource block allocation is GBR priority followed by non-GBR bearer services.

Table 1 Standardized QoS characteristics defined by 3GPP TS 23.203

\begin{tabular}{|l|l|l|l|l|l|}
\hline QCI & $\begin{array}{l}\text { Resource } \\
\text { type }\end{array}$ & Priority & $\begin{array}{l}\text { Packet } \\
\text { Delay }\end{array}$ & $\begin{array}{l}\text { Bit } \\
\text { Error }\end{array}$ & $\begin{array}{l}\text { Example } \\
\text { Service }\end{array}$ \\
\hline 3 & GBR & 2 & $100 \mathrm{~ms}$ & $10^{-2}$ & V2X Messages \\
\hline 8 & $\begin{array}{l}\text { Non } \\
\text { GBR }\end{array}$ & 8 & $300 \mathrm{~ms}$ & $10^{-6}$ & $\begin{array}{l}\text { TCP-Based } \\
\text { ftp, p2p etc. }\end{array}$ \\
\hline 80 & $\begin{array}{l}\text { Non } \\
\text { GBR }\end{array}$ & 6.8 & $10 \mathrm{~ms}$ & $10^{-6}$ & $\begin{array}{l}\text { Low Latency } \\
\text { eMBB }\end{array}$ \\
\hline 70 & $\begin{array}{l}\text { Non } \\
\text { GBR }\end{array}$ & 5.5 & $200 \mathrm{~ms}$ & $10^{-6}$ & $\begin{array}{l}\text { Mission } \\
\text { Critical Data: } \\
\text { www, Email, } \\
\text { ftp,p2p }\end{array}$ \\
\hline
\end{tabular}

When scheduling we consider packet delay budget. Overdue packets will be considered as invalid by GBR services. Therefore, GBR packets must have higher priority to acquire RBs. So, we say NonGBR packets are assigned lower priory as they have no critical demand on delay budget. We define a maximum bit rate to prevent starvation of resources from Non-GBR services.

\section{GAME THEORY AND ALGORITM DEVELOPMENT}

Game theory is the study of interactions between independent rational actors [25], [26]. The game consists of three core elements. 1. Players/Agents making decisions, 2. Actions/Choices that are available to the player, 3 . Payoffs/utilities received by the players after taking actions. In general game theory is split in to their cooperative or non-cooperative theory. For this work we are looking at cooperative wherein players form coalitions to improve their distributed payoffs. Within Cooperative game theory players are required to work together in coalitions to maximize payoffs. Given this our priority-based resource allocation is developed as a cooperative bargaining game wherein players Services/Slices choose strategies to maximize their own received resources via a group-based decision-making process. Resource provisioning is considered to by dynamic and therefore demand aware such that resources are allocated to 'users' based on requirement at a given instance.

Our proposed solution assigns resources to services according to their instantaneous minimum network requirement. We define our minimum network requirement as that which allows the service to operate within the designated QCI Packet Delay and Packet Error Loss definitions (Table 1) and the estimation of minimum network capacity for a service within a slice is given as:

$$
\begin{aligned}
& C_{i}=B_{i}\left(1+S N R_{i}\right) \\
& S N R_{i}=\frac{P}{N_{0} B_{i}}
\end{aligned}
$$

We utilize the Shannon Theorem stating that (1) determines the total capacity for user ' $\mathrm{i}$ ' for a given bandwidth $\mathrm{B}_{i}$. However, as we can see the channel capacity $\left(\mathrm{C}_{\mathrm{i}}\right)$ is dependent on the received bit energy to noise ratio (SNR). For our AWGN channel the capacity is given in Equation 2. Utilising the average signal power formula, we can obtain the Shannon capacity in terms of he bit energy and noise power special density given in Equation 4.

$$
C=B \log _{2}\left(1+\frac{P}{N_{o} B}\right)
$$

$$
C=B \cdot \log _{2}\left(1+\frac{E_{b}}{N_{0}} \cdot \frac{R}{B}\right)
$$

We rearrange Equation 4 to make Bandwidth B the subject:

$$
\begin{aligned}
& \text { Bandwidth : } R B_{A l} \cdot[12 \cdot S C S]
\end{aligned}
$$

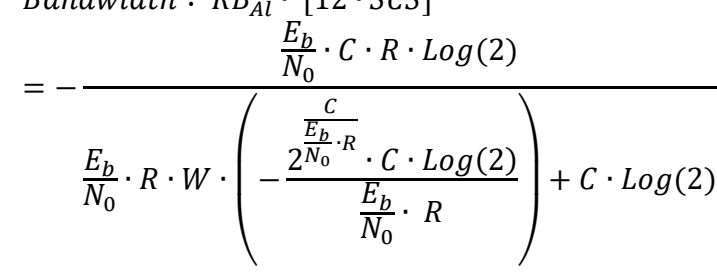

$\mathrm{C}=$ Projected minimum Network Capacity

$\mathrm{B}=$ Channel Bandwidth $(\mathrm{Hz})$

$\mathrm{P}=$ Constrained input signal power (watts)

$\mathrm{N}_{0}=$ one-sided noise power spectral density (watts/Hz)

$\mathrm{R}=1 / \mathrm{T}=$ Data/Transmission Rate (Bits/Second)

$\mathrm{RB}_{\mathrm{Al}}=$ Allocated Resource Blocks to a given service

SCS $=$ Sub-carrier Spacing

$\mathrm{E}_{\mathrm{B}}=$ Energy per data bit (Joules)

$\mathrm{E}_{\mathrm{B}} / \mathrm{N}_{0}=$ Received bit energy-to-noise spectral density ratio $\mathrm{W}=$ Product Logarithm

Equation 5 expresses bandwidth (allocated resource blocks multiplied by 12 x Sub-carrier Spacing) as a function of Network Capacity, Data Rate and SNR. Table 1 shows the QCI parameters for varying 3GPP use cases; for given network parameters we are able to define our received energy-to-noise spectral density to the appropriate QCI bit error rate.

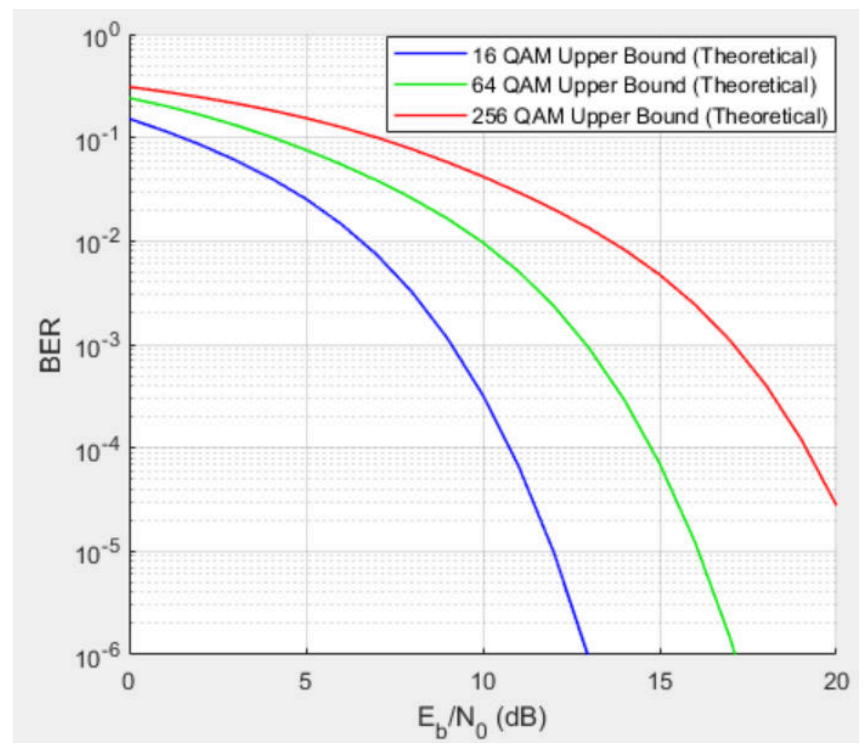

Figure 4 : Theoretical Maximum BER Vs. Eb/NO 
5G New Radio (NR) standard, utilizes OFDM for both uplink and downlink. The inherent flexibility of the physical layer gives a high degree of flexibility and therefore the ability to cover a wide range of applications; Carrier spacing is flexible up to 3300 subcarriers and the modulation can vary from QPSK, 16QAM, 64QAM or 256QAM. Greater orders of QAM result in higher throughput at the expense of noise resilience shown in Figure 4. For our simulation we have allocated $51 \mathrm{RBs}$ with $30 \mathrm{KHz}$ sub-carrier spacing for $20 \mathrm{MHz}$ bandwidth. Synchronized signal bursts have been ignored for this simulation.

Furthermore, a core requirement of the system is the satisfaction of an 'application defined' minimum data rate. Therefore, we substitute Equation 6 in to Equation 5:

$$
R=R_{S i, S} \cdot B_{S i, S}
$$

Where $\mathrm{R}_{\min }$ represents the minimum transmission rate that must be achieved to be within QoS compliance for a given service $S_{\mathrm{i}}$. B is a binary indicator to determine if bandwidth has been allocated to service $S_{i}$.

The problem of Resource Allocation within a multi-service scenario is formulated as a cooperative bargaining game. Therefore, we define the following:

- Players are given as $\mathrm{N}_{B}$ Services which are competing against each other for the remaining network Resource Blocks.

- A strategy which acts as our feasible solution where we give the amount of allocated resource blocks (ARB) to Service $s$ at instance $t_{i}$.

- The utility of service $\mathrm{N}_{B}$ which maps the given strategy onto a value in the range $[0,1]$, such that:

The utility of a service is proportional to the amount of the service demand that is being served; therefore, it is the service aim to maximize their utility function by joining the cooperative game.

There are three constraints that should be considered for the purpose of resource allocation. Firstly, the sum of RBs allocated across all services should not be more than Network RB Total. Secondly, for a given instance in time, the available network capacity for a service should not exceed the required service network capacity. Finally for the total amount of resource blocks enough must be reserved for SS burst transmissions - therefore our solution is bound within these constraints.

However, we realize that service priority is not uniform across all airlines or airports. Therefore, dynamic service QoS should be considered for resource allocation prioritization at the application layer once minimum QCI characteristics have been attained. To this end, each service is assigned a company determined weight at the application layer such that in the event that all services cannot be served to their maximum QoS at the same time the weights act as a priority indicator $(\sigma)$ (Equation 7) for additional resource allocation to active services:

$$
\sigma=\frac{P_{M a x}^{S_{i}}-P_{A l}^{S_{i}}}{P_{M a x}^{S_{i}}-P_{M i n}^{S_{i}}}
$$

- $P_{\text {Max }}^{S_{i}}$ : Maximum priority value available to be assigned at application layer to service $S_{i}$

- $\quad P_{A l}^{S_{i}}$ : Assigned priory value to service $S_{i}$

- $P_{\text {Min }}^{S_{i}}$ : Minimum priority value available to be assigned at application layer to service $S_{i}$
The resultant value is between 0 and 1 . A higher value of the priority indicator $(\sigma)$ provides a service a higher priority for radio resources. The solution we present utilizes the Generalized Nash Bargaining Solution Strategy to identify the distribution of resource among services to maximize resource utilization and QoS.

\section{General Nash Bargaining Solution:}

Finally, we propose a General Nash Bargaining Solution based on the sum of Equation 7 with the cooperative game utility function to allocate the remaining Physical Resource Blocks among the services weighted by priority.

\section{CONCLUSION}

In this paper we have explored aviation ground-ground services and potential communications solutions. Presented a high-level architecture for gNB infrastructure and proposed a novel algorithm based on cooperative game-theory that solves the resource allocation optimization and scheduling, and network QoS profile problems though a systematic selection of control variables (Resource Blocks). The model we have derived utilizes the strategic cooperation between services, slices, and resource blocks using the Shannon-Hartley theorem to express and allocate minimum required bandwidth for operation as a function of Received bit energy-tonoise spectral density ratio, Projected Capacity and Data Transmission rate. In addition, we have represented the problem of additional QoS Resource Allocation from the remaining resource pool within a multi-service scenario as a cooperative bargaining game and developed the initial framework for implementing flexible QoS assignment for aviation services. This has been presented in the form of General Nash Bargaining Solution which will be explored within a subsequent paper. We believe these algorithms could be used to maximize efficiency in 5G Ground/Ground networks and inter-slice resource coordination.

\section{ACKNOWLEDGMENT}

This project is supported by the grant received from Department for Transport (DfT), UK Government under the Future Aviation Security Solutions Industrial PhD Partnerships (FASS IPPs)). The research is carried out with the collaboration of Cranfield University, UK and Thales, UK. The authors of this publication are thankful to Thales, UK for providing funding and supporting this study.

\section{REFERENCES}

[1] M. Ehammer, T. Gräupl and E. Polo, "AeroMACS data traffic model," IEEE Integrated Communications, Navigation, and Surveillance Conference Proceedings, 2011

[2] Working Group C, Aeronautical Communications Panel, "An Introduction in AOC', Montreal, Canada, 2005

[3] ICAO Appendix I, Analysis of VHF Datalinks for Point-to-Point Communications with a focus on VDL Mode 4, 2009.

[4] VDL Mode 2 Capacity and Performance Analysis, SESAR, Nov 2015

[5] Challenges of Growth, task 4: European air traffic in 2035 (2013), EUROCONTROL

[6] M. J. Zeltser, F. R. Morser, P. Long, and F. Box, "White paper: Spectrum depletion analysis,'” The MITRE Corp., McLean, VA, USA, White Paper MP 03W0000068, 2003

[7] S. Ayub, I. Petrunin, A. Tsourdos, S. Al-Rubaye, G. Stapylton and G. Dent, "In-Flight Entertainment Datalink Analysis and Simulation," 2020 AIAA/IEEE 39th Digital Avionics Systems Conference (DASC), 2020

[8] S. Mondal, S. Al-Rubaye and A. Tsourdos, "Handover Prediction for Aircraft Dual Connectivity Using Model 
Predictive Control," in IEEE Access, vol. 9, pp. 44463-44475, 2021.

[9] A. Ahrens and B. Phillips, "Airport surface wireless technology and profile status," IEEE Integrated Communications, Navigation, and Surveillance Conference Proceedings, 2011

[10] D. Byrne, "AeroMACS Enabling Safe and Secure Connectivity Worldwide", WiMAX Forum, 2019

[11] G. Bartoli, R. Fantacci and D. Marabissi, "AeroMACS: A new perspective for mobile airport communications and services," IEEE Wireless Communications, vol. 20, no. 6, pp. 44-50, December 2013,

[12] S. Al-Rubaye, A. Al-Dulaimi, J. Cosmas and A. Anpalagan, "Call Admission Control for Non-Standalone 5G Ultra-Dense Networks," in IEEE Communications Letters, vol. 22, no. 5, pp. 1058-1061, May 2018.

[13] AeroMACS Aeronautical Mobile Airport Communication System: Standardized Soultion For the Airport Surface, A. Rocha , R. Simoes, WiMAX Forum, 2018

[14] S. Al-Rubaye and A. Tsourdos, "Airport Connectivity Optimization for 5G Ultra-Dense Networks," in IEEE Transactions on Cognitive Communications and Networking, vol. 6, no. 3, pp. 980-989, Sept. 2020

[15] A. Anand, G. De Veciana and S. Shakkottai, "Joint scheduling of URLLC and eMBB traffic in 5G wireless networks", Proc. IEEE Conf. Comput. Commun. (INFOCOM), pp. 1970-1978, Apr. 2018

[16] X. Foukas, G. Patounas, A. Elmokashfi and M. K. Marina, "Network slicing in 5g: Survey and challenges", IEEE Communications Magazine, vol. 55, no. 5, pp. 94-100, May 2017

[17] X. Foukas, G. Patounas, A. Elmokashfi and M. K. Marina, "Network Slicing in 5G: Survey and Challenges," in IEEE
Communications Magazine, vol. 55, no. 5, pp. 94-100, May 2017

[18] "An Introduction to Network Slicing" White Paper, GSM Association, 2017

[19] A. Papa, M. Klugel, L. Goratti, T. Rasheed and W. Kellerer, "Optimizing Dynamic RAN Slicing in Programmable 5G Networks," ICC 2019 - 2019 IEEE International Conference on Communications (ICC), 2019

[20] M. Pagin, F. Agostini, T. Zugno, M. Polese and M. Zorzi, "Enabling RAN Slicing Through Carrier Aggregation in mmWave Cellular Networks," 2020 Mediterranean Communication and Computer Networking Conference (MedComNet), 2020, pp. 1-8, doi: 10.1109/MedComNet49392.2020.9191462.

[21] N.Sharing, "Architecture and Function Description (Release 14)", 3gpp ts 23.251 v14 20170

[22] A. Al-Dulaimi, S. Al-Rubaye, J. Cosmas and A. Anpalagan, "Planning of Ultra-Dense Wireless Networks," in IEEE Network, vol. 31, no. 2, pp. 90-96, March/April 2017

[23] X. Yang, Y. Liu, K. S. Chou and L. Cuthbert, "A gametheoretic approach to network slicing," 2017 27th International Telecommunication Networks and Applications Conference (ITNAC), 2017

[24] 3GPP TS 28.531 Version 16.6.0 Release 16

[25] M. E. Mkiramweni, C. Yang, J. Li and W. Zhang, "A Survey of Game Theory in Unmanned Aerial Vehicles Communications," in IEEE Communications Surveys \& Tutorials, vol. 21, no. 4, pp. 3386-3416, Fourthquarter 2019,

[26] M. A. Arif, M. Ndoye, G. V. Murphy and K. Aganah, "A cooperative game theory algorithm for distributed reactive power reserve optimization and voltage profile improvement," 2017 North American Power Symposium (NAPS), 2017. 
$2021-11-15$

\section{Aircraft to operations communication analysis and architecture for the future aviation environment}

Whitworth, Huw

IEEE

Whitworth H, Al-Rubaye S, Tsourdos A, et al., (2021) Aircraft to operations communication analysis and architecture for the future aviation environment. In: 2021 AIAA/IEEE 40th Digital Avionics Systems Conference (DASC), 3-7 October 2021, San Antonio, New Mexico, USA https://doi.org/10.1109/DASC52595.2021.9594426

Downloaded from Cranfield Library Services E-Repository 